\title{
A Combination of Direct-to-Implant and Vertical Mastopexy Immediate Breast Reconstruction: A Case Report
}

\author{
Kwang Hyun Park, Bok Ki Jung, \\ Young Seok Kim, In Sik Yun, \\ Tai Suk Roh \\ Department of Plastic and Reconstructive \\ Surgery, Institute for Human Tissue \\ Restoration, Gangnam Severance \\ Hospital, Yonsei University College of \\ Medicine, Seoul, Korea
}

No potential conflict of interest relevant to this article was reported.
Patients with large or ptotic breasts who undergo nipple-sparing mastectomy and direct-to-implant (DTI) reconstruction tend to exhibit poor esthetic outcomes. We present a combined vertical mastopexy-DTI technique that can be used to raise the nipple position and to improve esthetic outcomes. Using the combined vertical mastopexyDTI technique, it is possible to save time and to increase patient satisfaction.

Keywords Breast implant, Breast neoplasm, Breast reconstruction, Mammaplasty, Reduction

\section{INTRODUCTION}

Recently, many studies have demonstrated that nipple-sparing mastectomy (NSM) does not increase the risk of tumor recurrence [1-3]. In light of the advantages of immediate breast reconstruction and improvements in techniques such as the use of acellular dermal matrix (ADM), many patients have successfully undergone NSM and direct-to-implant (DTI) reconstruction [4].

In our hospital, preoperative planning for NSM includes an inframammary fold (IMF) approach to preserve sufficient blood flow to the nipple-areolar complex [5]. However, patients with large or ptotic breasts who undergo NSM and DTI reconstruction tend to exhibit poor esthetic outcomes. We present a combined vertical mastopexy-DTI technique that can be used to raise the

Received: Mar 21, 2018 Revised: Apr 18, 2018 Accepted: Apr 19, 2018 Correspondence: Tai Suk Roh Department of Plastic and Reconstructive Surgery, Gangnam Severance Hospital, Yonsei University College of Medicine, 211 Eonju-ro, Gangnam-gu, Seoul 06273, Korea.

E-mail: ROHTS@yuhs.ac

Copyright @ 2018 The Korean Society for Aesthetic Plastic Surgery.

This is an Open Access article distributed under the terms of the Creative Commons Attribution Non-Commercial License (http://creativecommons.org/licenses/by-nc/4.0/) which permits unrestricted non-commercial use, distribution, and reproduction in any medium, provided the original work is properly cited. $\quad w w w . e-a a p s . o r g$ nipple position and to improve esthetic outcomes.

\section{Surgical technique}

The NSM incision design and planning of the IMF approach were done on the day before surgery. The technique was divided into 2 steps. First, NSM was performed via a vertical incision (nipple to IMF). After NSM, ADM was fixed to the inferior part of the breast and an adequate-sized implant was selected according to the specimen weight and inserted into the subpectoral and ADM pocket via a vertical incision. Then, the patient was placed in a $90^{\circ}$ sitting position and the distance from the nipple to the suprasternal notch was measured. The new nipple location was marked and placed $23.5 \mathrm{~cm}$ from the suprasternal notch. A vertical mastopexy was designed on this basis (Fig. 1). De-epithelization was performed on the newly formed nipple-areolar complex site, and skin was removed along the vertical incision margin. We determined the amount of skin to be removed by placing the patient in the sitting position to check the skin redundancy and tension.

\section{CASE REPORT}

The patient was a 45-year-old woman with moderately sized and moderately ptotic breasts (grade II), who had recurrent invasive ductal carcinoma in the upper medial quadrant of the left breast. 

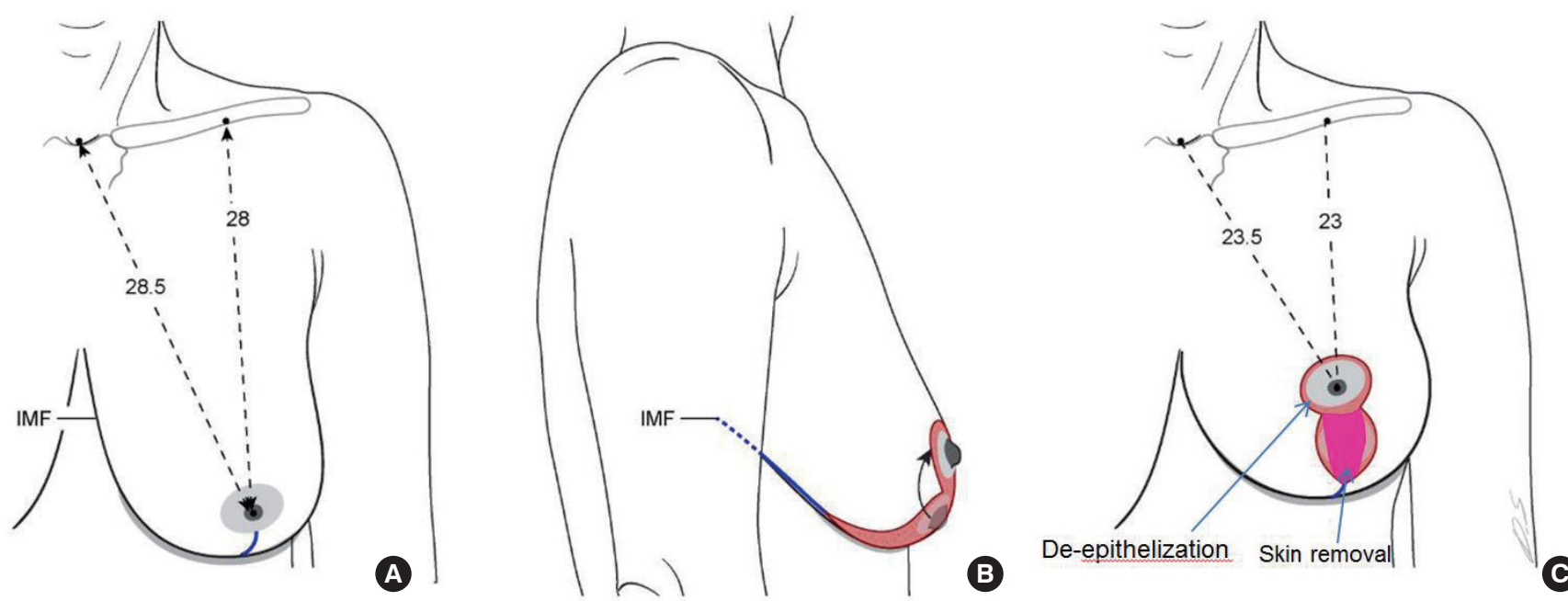

Fig. 1. (A) The preoperatively designed vertical incision (blue line). The oncologic surgeon performed nipple-sparing mastectomy via this incision. (B) Lateral intraoperative view. The red line is the vertical mastopexy design. (C) Anteroposterior intraoperative view. The area with red dots is the de-epithelization area and the pink areas are the skin removal site.

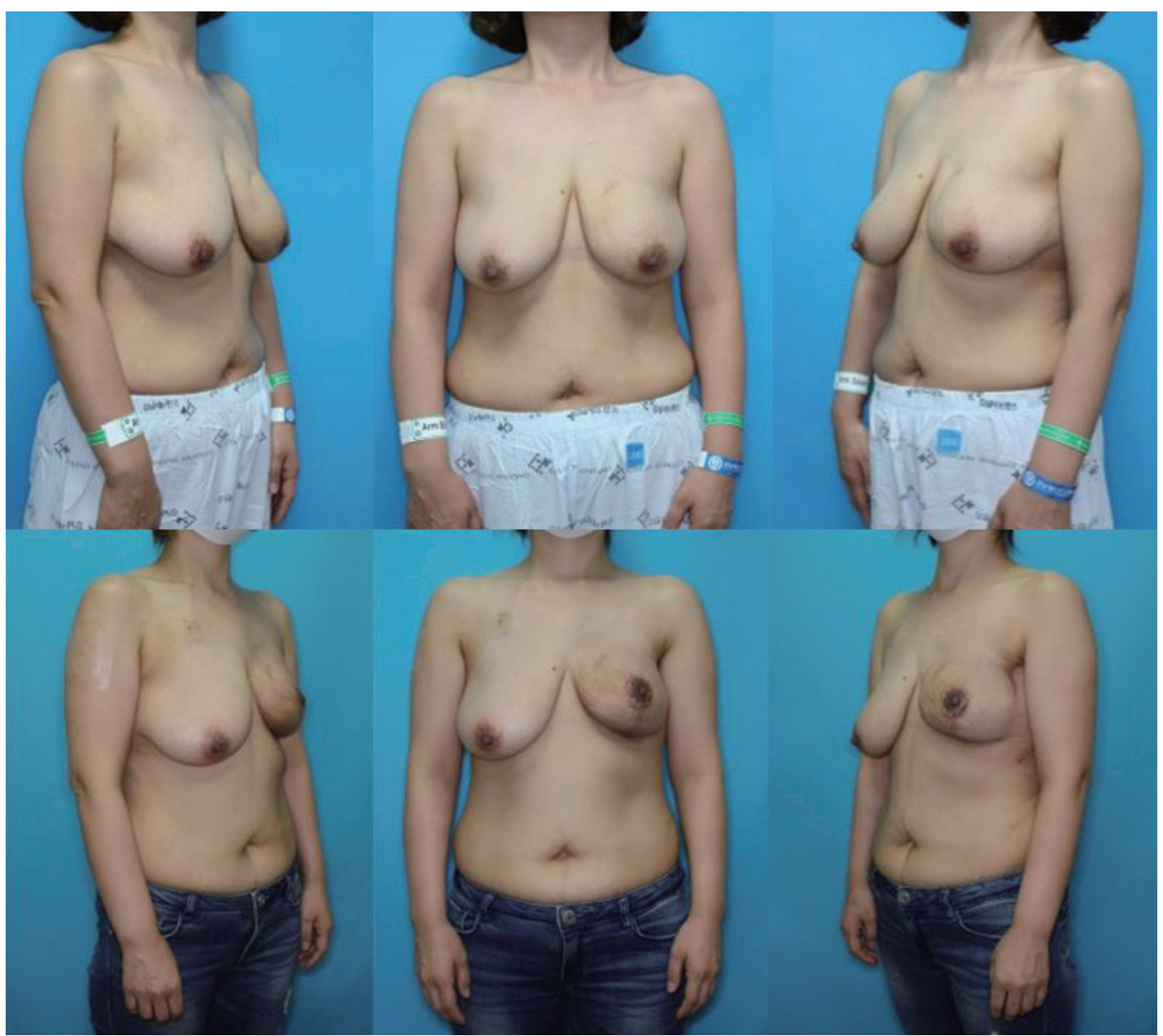

Fig. 2. Preoperative frontal and semi-lateral views (above). Frontal and semi-lateral view 3 months after immediate breast reconstruction using a combination of direct-to-implant reconstruction and vertical mastopexy (below). 
The patient had undergone partial mastectomy and adjuvant radiation therapy 7 years prior. Upon recurrence, the patient underwent NSM through a vertical incision, following our design. The specimen weight was $491 \mathrm{~g}$. After NSM, a permanent implant (CPG 311 Mentor $^{\circledR} 270 \mathrm{~mL}$; Mentor Corporation, Santa Barbara, CA, USA) was inserted under the pectoralis major muscle and ADM pocket. Then, the patient was moved into a $90^{\circ}$ sitting position and vertical mastopexy was done to place the nipple $23.5 \mathrm{~cm}$ from the suprasternal notch. The operating time was 86 minutes. The cosmetic result was good and the patient's satisfaction was very high. Adjuvant chemotherapy was recommended because the patient had triple-negative disease. The patient requested contralateral mastopexy after chemotherapy (Fig. 2).

\section{DISCUSSION}

It is a challenge for plastic surgeons to reconstruct large, ptotic breasts using implants or expanders. Many plastic surgeons recommend autologous reconstruction procedures, such as transverse rectus abdominis flaps, in these cases [4]. In such cases, the goal is not only to reduce the operating time and to minimize scarring, but also to correct ptotic breasts.

Appropriate blood supply to the skin flap is also a major issue in these procedures. Hudson and Skoll [6] reported that they performed DTI reconstruction after mastectomy using a Wise-pattern mastopexy design. The total complication rate (skin necrosis) was 3 of 19 (15.7\%). Hammond et al. [7] reported using a tissue expander after mastectomy with a Wise-pattern design. The total complication rate (skin necrosis) was 2 of 12 (16.6\%). In mastectomy with a Wisepattern design, preventing skin flap necrosis is a challenge [6,7]. Thus, we performed DTI reconstruction because there are no major differences in complication rates between tissue expanders and DTI reconstruction. To preserve adequate blood supply for the skin flap, we used a vertical mastopexy design rather than a Wise-pattern mastopexy design. Additionally, a 2-stage design can help ensure adequate size of the implant so that it conforms to the skin tension.

Preserving a large dermoadipose flap can reduce the skin flap necrosis rate. Algaithy et al. [8] reported that after mastectomy, a remaining dermoadipose flap thickness of less than $5 \mathrm{~mm}$ was a significant risk factor of necrosis. In this context, a "large" dermoadipose flap refers precisely to a thickness of more than $5 \mathrm{~mm}$. Thus, preoperative consultation with the oncologic surgeon and preservation of the dermoadipose flap within an oncologically safe range would be ideal [9].

In our institution, DTI reconstruction takes 70 minutes on average. The entire reconstruction procedure takes only 86 minutes. The reconstructive effectiveness of this technique was confirmed in terms of operating time and patient satisfaction (very high). The contralateral side can be reconstructed at the same time for symmetry, or the contralateral reconstruction can be done later, as in this case.

\section{CONCLUSIONS}

We present an innovative method for the reconstruction of large ptotic breasts. Using the combined DTI and vertical mastopexy technique, it is possible to save time and to increase patient satisfaction. Skin flap necrosis is a serious complication, but it can be avoided by using an adequately sized implant, performing verticalpattern mastopexy rather than Wise-pattern mastectomy, and preserving a large dermoadipose flap.

\section{PATIENT CONSENT}

Patient provided written consent for the use of her images.

\section{REFERENCES}

1. Maxwell GP, Storm-Dickerson T, Whitworth P, et al. Advances in nipple-sparing mastectomy: oncological safety and incision selection. Aesthet Surg J 2011;31:310-9.

2. Radovan C. Breast reconstruction after mastectomy using the temporary expander. Plast Reconstr Surg 1982;69:195-208.

3. Singletary SE. Skin-sparing mastectomy with immediate breast reconstruction: the M. D. Anderson Cancer Center experience. Ann Surg Oncol 1996;3:411-6.

4. Breuing KH, Colwell AS. Inferolateral AlloDerm hammock for implant coverage in breast reconstruction. Ann Plast Surg 2007;59:250-5.

5. Colwell AS, Damjanovic B, Zahedi B, et al. Retrospective review of 331 consecutive immediate single-stage implant reconstructions with acellular dermal matrix: indications, complications, trends, and costs. Plast Reconstr Surg 2011;128:1170-8.

6. Hudson DA, Skoll PJ. Complete one-stage, immediate breast reconstruction with prosthetic material in patients with large or ptotic breasts. Plast Reconstr Surg 2002;110:487-93.

7. Hammond DC, Capraro PA, Ozolins EB, et al. Use of a skin-sparing reduction pattern to create a combination skin-muscle flap pocket in immediate breast reconstruction. Plast Reconstr Surg 2002;110:206-11.

8. Algaithy ZK, Petit JY, Lohsiriwat V, et al. Nipple sparing mastectomy: can we predict the factors predisposing to necrosis? Eur J Surg Oncol 2012;38:125-9.

9. Nava MB, Ottolenghi J, Pennati A, et al. Skin/nipple sparing mastectomies and implant-based breast reconstruction in patients with large and ptotic breast: oncological and reconstructive results. Breast 2012; 21:267-71. 